\title{
Takotsubo Cardiomyopathy presenting as ST-elevation myocardial infarction: wide triggering spectrum and specific echocardiographical pattern in a consecutive case series report
}

\author{
Călin Homorodean ${ }^{1,2}$, Mihai C. Ober², Maria Olinic², Romana Homorodean², \\ Abdesamad Hassoune², Dan Tătaru' ${ }^{1}$, Mihai Spînu', Dan M. Olinic1, 2
}

${ }^{1} 1$ st Medical Department, "Iuliu Hatieganu” University of Medicine and Pharmacy, ${ }^{2}$ Interventional Cardiology Department, Emergency County Hospital, Cluj-Napoca, Romania

\begin{abstract}
We report a series of six consecutive cases of Takotsubo Cardiomyopathy (TCM) presenting as ST-elevation acute myocardial infarction and consequently addressed to the emergency coronary angiography, inside a time-frame of one year (2015). This series underlines the wide possible triggering spectrum and clinical severity of TCM and recognizes echocardiography as an essential imaging technique in the diagnosis due to its widespread availability and feasibility in the acute care setting.

Keywords: Takotsubo cardiomyopathy, wall motion abnormalities, echocardiography
\end{abstract}

\section{Introduction}

An association between emotional or physical stressful triggers and cardiovascular adverse events has been described for many years.

Transient left ventricular (LV) apical ballooning is a cardiac syndrome characterized by LV dysfunction accompanied by echocardiographic, electrocardiographic, and biologic features mimicking an acute myocardial infarction [1], usually preceded by emotional or physical stressors. It was first described in Japan and it was named Takotsubo cardiomyopathy (TCM) because of the resemblance between the left ventricular appearance in systole and the shape of a pot with a round bottom and a narrow neck (Takotsubo $=$ Japanese octopus trap) [2,3]. This syndrome is characterized by acute LV dysfunction with regional wall motion abnormalities (WMA) extended beyond a single

Received 07.07.2016 Accepted 14.08.2016

Med Ultrason

2016, Vol. 18, No 4, 475-480

Corresponding author: Maria Olinic $\mathrm{MD}, \mathrm{PhD}$

$1^{\text {st }}$ Medical Department, "Iuliu Hatieganu

"University of Medicine and Pharmacy

3-5, Clinicilor street,

400006 Cluj-Napoca, Romania

Phone: + 40745522 781, Fax: +40 264596400

E-mail: maria_olinic@yahoo.com coronary vascular bed, absence of significant obstructive coronary obstructive disease, and rapid improvement of LV function within a few days or weeks [4].

TCM has been recognized as an important consideration in the differential diagnosis of acute coronary syndromes [5]. The difference with anterior myocardial infarction accounts for the extent of LV dysfunction, far beyond left anterior descending artery (LAD) territory [4]. The prevalence of this pathology is estimated to be $2 \%$ of patients undergoing coronary angiography for acute coronary syndromes [6,7].

In the specific settings of acute interventional management of ST-elevation myocardial infarction (STEMI), TCM recognition represents a rare but important finding since these patients have the potential to completely recover if they are properly supported to overcome the acute phase [8]. Therefore, we report the consecutive cases of TCM encountered during one year of STEMI interventional management in a high-experienced center.

During the year 2015 we performed emergency coronarography in 258 patients with STEMI within the first 12 hours of onset and we diagnosed TCM in 6 patients $(2.3 \%)$. We used a modified version of Mayo Clinic Criteria for Diagnosis of TCM [9], including also the patients who developed TCM in the setting of neurological disorders [10]. 


\section{Case 1}

A 57 year old male patient, with epilepsy, was admitted with an anterior STEMI complicated with cardiogenic shock. Chest pain was preceded by two seizures. Initial ECG showed minimal ST elevation in the precordial leads. Echocardiographic findings consisted of circumferential mid-ventricular akinesia and apical dyskinesia, a Wall Motion Abnormality Scoring Index (WMSI) of 2.35 and a severely depressed LV ejection fraction (EF) of $20 \%$ (fig 1a,b). Troponin levels were only slightly elevated, disproportionate to the large WMA. Coronarography revealed normal coronary arteries. Intra-aortic balloon pumping (IABP) was required during the first 3 days. In the 15th day, echo control revealed significantly improved EF (45\%) with apical hypokinesia. He was discharged the $16^{\text {th }}$ day. One month later his EF was normal without wall motion abnormalities (fig 1c,d). We interpreted the case as TCM induces by seizures.

\section{Case 2}

A 73 year old female patient presented to the emergency room with rest chest pain associated with dyspnea. The patient had a severe psychological impact several days before hospitalization. On admission, she had stable blood pressure levels but signs of acute left ventricular failure were present. Initial evaluation revealed, ST-elevation in DI, aVL on ECG, slightly increased Troponin levels, akinesia in the apical segments with WMSI of 1.85 and $\mathrm{EF}$ of $35 \%$. Coronary arteries were normal. A followup echocardiogram performed in the $5^{\text {th }}$ day of hospitalization, revealed marked improvement in WMA with only mild apical hypokinesia and normal EF. This case was regarded as a TCM with a psycho-emotional trigger.

\section{Case 3}

A 80 year old female presented in the emergency ward after a syncopal episode. She had a recent history of interstitial pneumonia. Initial ECG showed ST-elevation in the D I and aVL leads and troponin levels were elevated. Echocardiography revealed a LV with severe hipokinesia in the apical segments (WMSI $=1.25$ ) with preserved EF. Emergency coronary angiography revealed normal coronary arteries. During the second day of hospitalization she developed three episodes of "torsade de pointes" on the background of a QT-segment elongation, requiring electric conversion. ECG and echocardiogram aspects entirely normalized until the 10th day of hospitalization. The patient met the criteria for TCM though the triggering factor was not entirely clear (possible stress induced by physical illness: pneumonia).

\section{Case 4}

A 55 year old female, developed a clinical picture of STEMI complicated with cardiogenic shock in the sec-

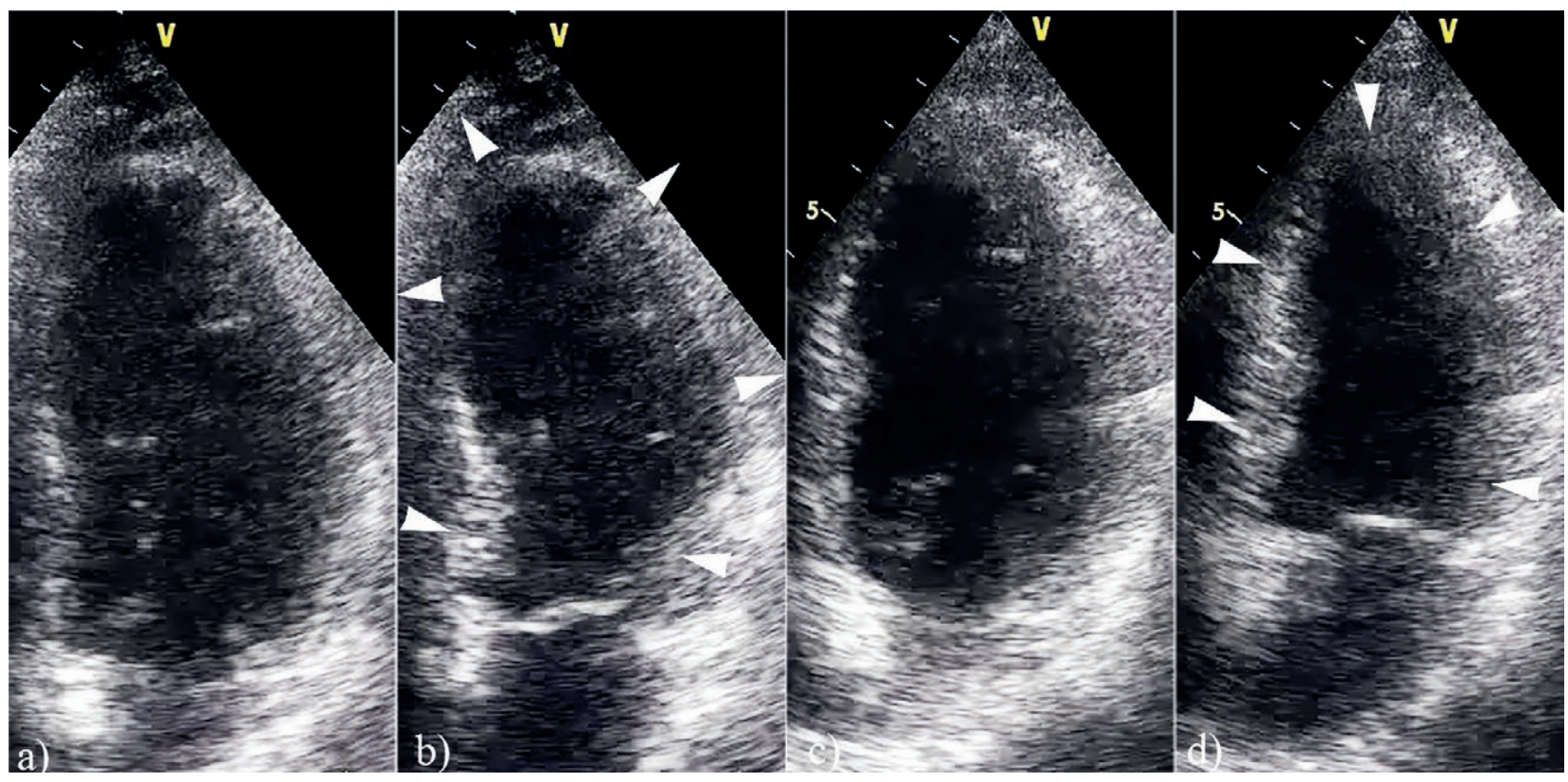

Fig 1. Echocardiography in a patient with TCM induced by seizures: a) Apical four chambers echocardiograms recorded during acute phase in diastole and, b) in systole, revealing apical ballooning; c) Echocardiograms recorded after one month, in diastole and, d) in systole, showing complete recovery. Arrowheads highlight wall motion direction. 
ond day after a neurosurgical intervention for a cerebral aneurysm, with consecutive subarachnoid hemorrhage (SAH). Blood pressures of $80 \mathrm{mmHg}$ were maintained under inotrop infusion. Echocardiography revealed large akinesia involving all mid ventricular segments, apical diskinesia and EF of $20 \%$. Moreover, WMA involved also the right ventricle with a severely decreased tricuspid annular plane systolic excursion (TAPSE). Coronarography showed normal coronary arteries. After 24 hours she died due to hemodynamic instability. The suspicion
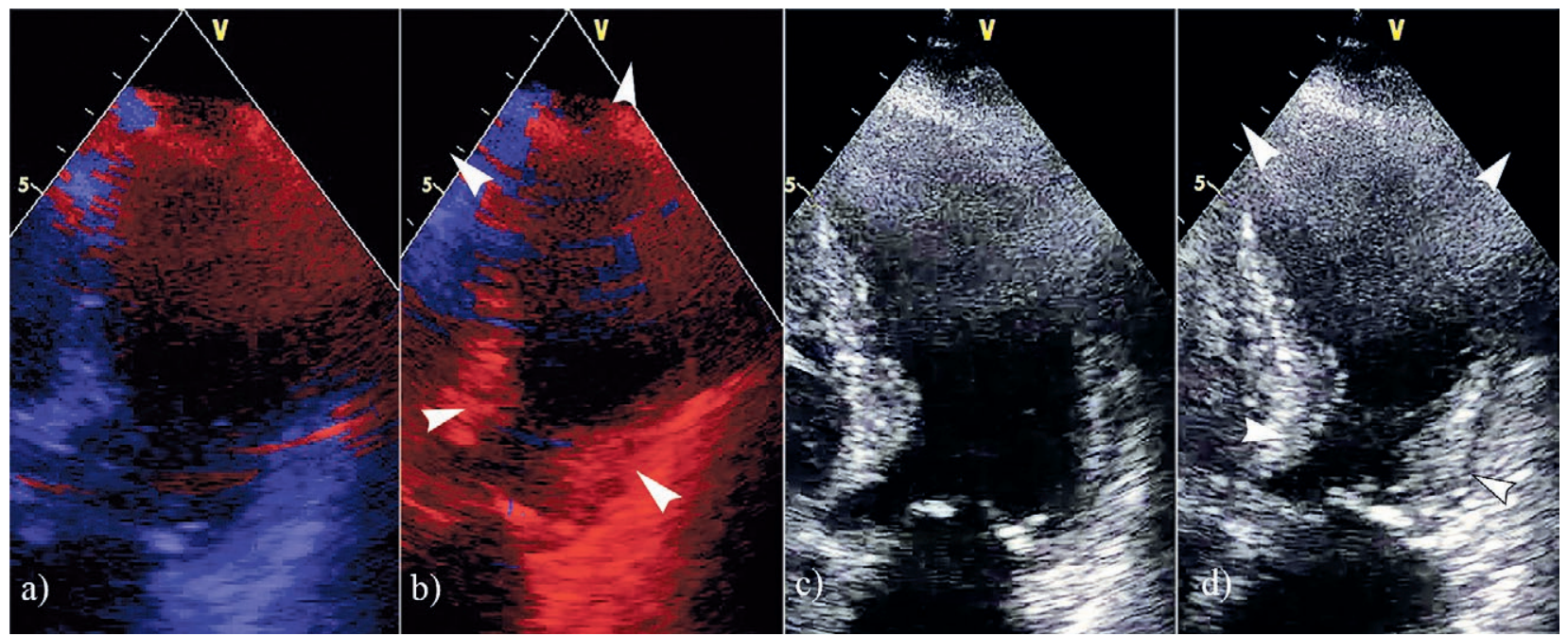

Fig 2. TCM triggered by emotional stress induced by severe epistaxis (acute phase): a) Color Tissue-Doppler imaging recordings in diastole and, b) in systole; 2D echocardiograms are depicted in panels c) during diastole and d) systole. Arrowheads underlie wall motion direction

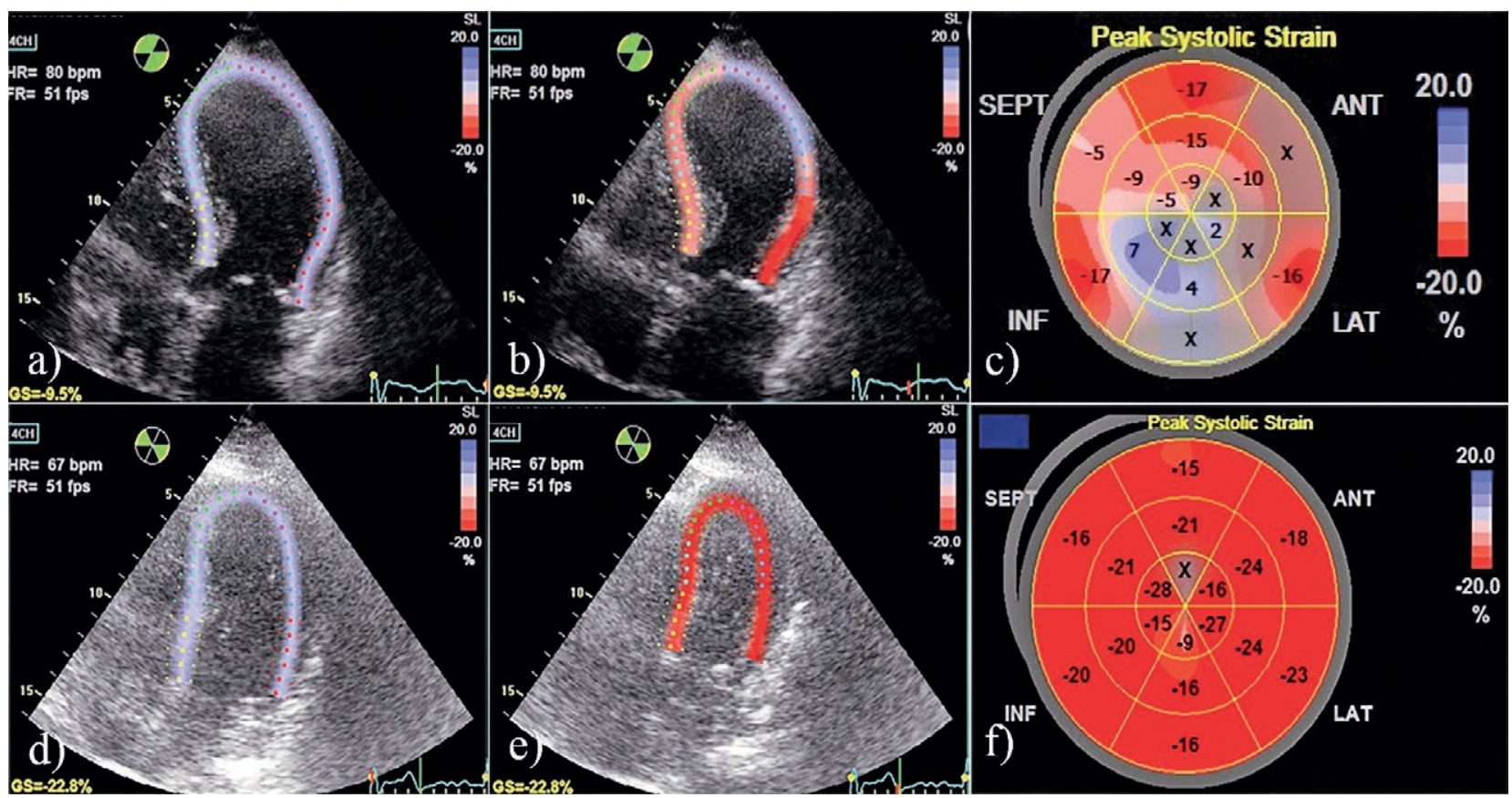

Fig 3. Longitudinal strain (LS) imaging in a patient with TCM triggered by epistaxis. Acute phase: a) End-diastolic LS in A4Ch view; b) Peak instantaneous LS during systole in A4Ch view, showing basal left ventricular systolic shortening (red = negative LS) and apical systolic lengthening (blue = positive LS); c) Bull's Eye plotting of the peak systolic strain from PLAX, A4Ch and A2Ch views. At 6-months follow-up showing complete recovery: d) End-diastolic LS in A4Ch view; e) Peak instantaneous LS in A4Ch view, revealing homogeneous shortening (red) consecutive to complete recovery of myocardial kinetics; f) Bull's Eye plotting of peak systolic strain from PLAX, A4Ch and A2Ch views. PLAX = parasternal long axis view, A4Ch= Apical four chambers view, A2Ch=Apical two Chambers view. 
of TCM induced by SAH (by Mayo Clinic criteria) [10] was raised, though the criteria of LV function improvement was not met. According to the Japanese Guidelines for diagnosis of TCM proposed in 2007, this patient would have been diagnosed as "cerebrovascular disease with Takotsubo-like myocardial dysfunction" [11,12].

\section{Case 5}

An 80 year old female was admitted with anterior STEMI suspicion. Clinical picture of STEMI developed after an episode of epistaxis which triggered a great emotional impact. Ultrasound examination revealed a LV apical ballooning pattern both on color Tissue-Doppler imaging (fig 2a,b) and 2D echo-mode (fig 2c,d), with EF of $40 \%$.

Two-dimensional longitudinal strain was performed (fig 3a,b,c). A severely decreased global longitudinal strain was measured during the acute phase of TCM. Longitudinal strain detected altered LV mechanics also at the base of the heart. No coronary artery lesions were found. Echocardiographic control a week later revealed LV wall motion normalization with a normal global longitudinal strain (fig 3d,e,f).

\section{Case 6}

A 74 year old female patient presented with severe chest pain and dyspnea triggered by a nightmare with severe emotional discomfort. ECG revealed ST-segment elevation in V1-V2 and deep large $\mathrm{T}$ waves in V3-V6 leads. An emergency coronary angiography was performed, with normal coronary arteries. Echocardiography revealed apical hypokinesia with a WMSI of 1.25 and preserved global LV ejection fraction. Control echocardiography performed in the $3^{\text {rd }}$ day of hospitalization showed complete LV recovery. The case was interpreted as emotionally induced TCM.

\section{Discussions}

TCM is a rather rare pathology though its prevalence may be underestimated either by under-recognition of the less severe TCM cases or by non-inclusion of the patients who die acutely [13]. Moreover, the criteria we used, addressed only to cases of STEMI with normal coronary artery disease. However, latest recent papers also recognize a "TCM component" in cases of myocardial infarction with significant coronary lesions [13]. Therefore, considering the aforementioned data, in daily practice, TCM prevalence might be much greater than currently presented in the literature (1.7-2.2\%) [1] or observed in our center $(2.3 \%)$.
Prognosis of TCM is generally favorable, but a small subset has potentially life-threatening complications [12]. In a very large study, including all TCM patients from US hospitals during 2008-2009, in-hospital mortality was $4.2 \%$ whereas cardiogenic shock was present in $4.7 \%$ of cases [14].

Regarding the triggering factors, a preceding acute medical condition induced TCM in $43 \%$ of cases and emotional factors in the remaining cases [2]. Our case series illustrates the very heterogeneous nature of the clinical presentations, triggering factors and outcomes severity in TCM. The two patients with TCM induced by neurological causes (epilepsy and SAH) had a very severe course: both patients had extensive WMA, both developed cardiogenic shock and the patient with SAH died during the first 48 hours of onset. The severe outcomes of neurogenic TCM have been already demonstrated in previous papers. In the study of Stollberger et al [15], patients with TCM induced by seizures had a more serious course when compared to other TCM patients. Seizure-associated TCM manifests frequently as sudden hemodynamic deterioration which could result in death. Catecholamine release due to seizures might be more excessive and last longer, compared with other emotional or physical triggers of TCM [15]. Additionally, patients with SAH and TCM are known to have a very severe outcome, with a mortality of $36.6 \%$ in series of Kent et al [16].

Echocardiography is the essential imaging technique in the diagnosis of TCM and in its differentiation from acute myocardial infarction and include both classical techniques (as WMA evaluation by 2D-echo mode) and advanced techniques (as myocardial deformation imaging - longitudinal and radial strain). In the TCM patients, WMA extended beyond one coronary bed (e.g. LAD territory). Due to technical reasons we performed advanced myocardial deformation techniques only in a single patient. Due to its higher sensitivity in detecting subtle abnormalities when compared to the more traditional parameters like EF or WMSI, longitudinal strain detected altered LV mechanics also at the base of the heart. There are studies that showed that myocardial impairment in TCM extends beyond the segments with classical WMA [4] and that longitudinal strain in some of the posterior, inferior, and lateral segments is significantly lower in TCM compared to STEMI [17]. Moreover, when compared to healthy individuals, in TCM patients, longitudinal strain is diminished in almost all segments and not only in the anterior ones [17].

It is known that right ventricular dysfunction is a marker of the severity of TCM and is associated with lower LV EF [18]. The most severe patient (TCM in- 
duced by SAH) of our case series, also had right ventricular WMA.

Some interactions between TCM and medication used in STEMI patients are worth mentioning. There are reports on the TCM development in patients receiving drugs such as epinephrine, even after exposure to doses routinely used in clinical practice [19]. We may speculate that catecholamine treatment in patients with STEMI and cardiogenic shock could induce in some patients a superimposed TCM, with very deleterious effects. Conversely, catecholamine-type inotropes administration in TCM complicated by cardiogenic shock have the potential of further aggravating the clinical course. Therefore, in these patients mechanical assistance or non-catecholamine inotropes such as levosimendan should be favored [8].

The mechanisms underlying TCM are not entirely known but a catecholamine surge was suggested [5]. High levels of circulating epinephrine may trigger in ventricular cardiomyocyte a switch in intracellular signaling from Gs to Gi protein, via beta 2 adrenoceptor (AR) [20]. At normal values, epinephrine binds to beta 2 AR resulting in positive inotropic effects through a $\mathrm{Gq}$ protein signaling. At higher concentrations, epinephrine stimulates a negative inotropic effect on myocites by $\mathrm{Gi}$ protein [20]. It was demonstrated that elevated catecholamine levels decrease the viability of myocytes through cyclic AMP-mediated calcium overload [12].

\section{Conclusion}

This case series highlights the wide spectrum of possible triggering factors and clinical severities of TCM that are encountered by the interventional cardiologist in the real-life STEMI management. It also underlines the echocardiography as an essential imaging technique in the TCM diagnosis due to its widespread availability and feasibility in the acute care setting. Furthermore, some of the cases presented are an illustration of the fact that rapid diagnosis of TCM complicated by cardiogenic shock sets in motion specific treatment strategies (preferential use of intra-aoric balloon pumping over inotropic drugs).

\section{References}

1. Gianni M, Dentali F, Grandi AM, Sumner G, Hiralal R, Lonn E. Apical ballooning syndrome or takotsubo cardiomyopathy: a systematic review. Eur Heart J 2006;27:15231529.

2. Kawai S, Suzuki H, Yamaguchi H, et al. Ampulla cardiomyopathy ('Takotusbo' cardiomyopathy) - reversible left ventricular dysfunction: with ST segment elevation. Jpn Circ J 2000;64:156-159.

3. Dote K, Sato H, Tateishi H, Uchida T, Ishihara M. Myocardial stunning due to simultaneous multivessel coronary spasm: a review of 5 cases. J Cardiol 1991;21:203-214.

4. Citro R, Lyon AR, Meimoun P, et al. Standard and advanced echocardiography in Takotsubo (stress) cardiomyopathy: clinical and prognostic implications. J Am Soc Echocardiogr 2015;28:57-74.

5. Hurst RT, Prasad A, Askew JW 3rd, Sengupta PP, Tajik AJ. Takotsubo cardiomyopathy: a unique cardiomyopathy with variable ventricular morphology. JACC Cardiovase Imaging 2010;3:641-649.

6. Bybee KA, Prasad A, Barsness GW, et al. Clinical characteristics and thrombolysis in myocardial infarction frame counts in women with transient left ventricular apical ballooning syndrome. Am J Cardiol 2004;94:343-346.

7. Parodi G, Del Pace S, Carrabba N, et al. Incidence, clinical findings, and outcome of women with left ventricular apical ballooning syndrome. Am J Cardiol 2007;99:182-185.

8. Lyon AR, Bossone E, Schneider B, et al. Current state of knowledge on Takotsubo syndrome: a Position Statement from the Taskforce on Takotsubo Syndrome of the Heart Failure Association of the European Society of Cardiology. Eur J Heart Fail 2016;18:8-27.

9. Prasad A. Apical ballooning syndrome: an important differential diagnosis of acute myocardial infarction. Circulation 2007; 115:e56-e59.

10. Prasad A, Lerman A, Rihal CS. Apical ballooning syndrome (Tako-Tsubo or stress cardiomyopathy): a mimic of acute myocardial infarction. Am Heart J 2008; 155:408-417.

11. Kawai S, Kitabatake A, Tomoike H; Takotsubo Cardiomyopathy Group. Guidelines for diagnosis of Takotsubo (ampulla) cardiomyopathy. Circ J 2007:71;990-992.

12. Ono R, Falcão LM. Takotsubo cardiomyopathy systematic review: Pathophysiologic process, clinical presentation and diagnostic approach to Takotsubo cardiomyopathy. Int J Cardiol 2016;209:196-205.

13. Madias JE. Why the current diagnostic criteria of Takotsubo syndrome are outmoded: A proposal for new criteria. Int J Cardiol 2014;174:468-470.

14. Brinjikji W, El-Sayed AM, Salka S. In-hospital mortality among with takotsubo cardiomyopathy: a study of the National Inpatient Sample 2008 to 2009. Am Heart J 2012;164:215-221.

15. Stollberger C, Wegner C, Finsterer J. Seizure-associated Takotsubo cardiomyopathy. Epilepsia 2011;52:e160-e167.

16. Kilbourn KJ, Ching G, Silverman DI, McCullough L, Brown RJ. Clinical outcomes after neurogenic stress induced cardiomyopathy in aneurysmal sub-arachnoid hemorrhage: A prospective cohort study. Clin Neurol Neurosurg 2015;128:4-9.

17. Heggemann F, Hamm K, Kaelsch T, et al. Global and regional myocardial function quantification in Takotsubo cardiomyopathy in comparison to acute anterior myocardial infarction using two-dimensional (2D) strain echocardiography. Echocardiography 2011;28:715-719. 
18. Kato K, Kitahara H, Fujimoto Y, et al. Prevalence and Clinical Features of Focal Takotsubo Cardiomyopathy. Circ J 2016;80:1824-1829.

19. Abraham J, Mudd JO, Kapur NK, Klein K, Champion HC, Wittstein IS. Stress cardiomyopathy after intravenous administration of catecholamines and beta-receptor agonists. J Am Coll Cardiol 2009;53:1320-1325.
20. Lyon AR, Rees PS, Prasad S, Poole-Wilson PA, Harding SE. Stress (Takotsubo) cardiomyopathy-a novel pathophysiological hypothesis to explain catecholamine-induced acute myocardial stunning. Nat Clin Pract Cardiovasc Med 2008;5:22-29. 